\title{
Comparison of Binning and Sorting of Magnets in the SSC High Energy Booster*
}

Mingyang $\mathrm{Li}^{\dagger}$ and Shoroku Ohnuma

Physics Department, University of Houston

Houston, Texas 77204-5506

\section{Abstract}

Two schemes for enlarging the usable aperture in superconducting synchrotrons, the binning of dipoles with several independent correction circuits and the sorting of dipoles based on their random sextupole components, are investigated for the SSC High Energy Booster by tracking a particle for two-thousand turns. With the anticipated $\mathrm{rms}$ values of $b_{2}$ and $\mathrm{a}_{2}$ when the coil-winding diameter is $5 \mathrm{~cm}$, we have found that the sorting is comparable in overall effectiveness as the binning with six independent circuits which will effectively reduce the rms value by a factor of four.

\section{INTRODUCTION}

It is by now well-established that the dominant factor in limiting the dynamic aperture (for a few thousand to a few tens of thousands turns) in superconducting synchrotrons is the random sextupole field in dipole magnets. So far, two schemes have been proposed to enlarge the usable aperture: one called "binning" is to have trim sextupole windings which will be connected to several independent circuits [1], and the other called "sorting" is to make the optimum arrangement of a given set of dipoles in the ring. A simple procedure for finding the optimum arrangement has been proposed by Gluckstern and Ohnuma [2] and its effectiveness has been investigated for various cases $[3,4]$.

With six circuits for binning, one can expect that $\mathrm{rms}$ value of random sextupole component will be effectively reduced by a factor of four and the effectiveness of the correction will not be affected by any other factors. This is a definite advantage of binning over sorting since the latter is based on the expected reduction in certain harmonic components of the random field rather than the random field itself. As such, the effectiveness of sorting in enlarging the dynamic aperture may be adversely affected by a variety of factors such as the closed orbit distorions, change in tunes from the design

\footnotetext{
- Work supported by the U.S. Department of Energy under grant number DE-FG05-87ER40374.

† Present address: MS 1049. The SSC Laboratory, Dallas, Texas, 75237
}

values, orbit distortion due to the momentum spread in the beam, and the lattice chromaticities. It is also obvious that the sorting will be less effective as the betatron phase advance over one dipole increases.

A question on the required dynamic aperture of the SSC High Energy Booster (HEB) and the closely related issue of the coil-winding diameter of its dipoles have been raised recently by members of the SSC Laboratory [5]. Because of the decision to increase the diameter of the Collider dipoles to $5 \mathrm{~cm}$, it is natural to ask whether one can provide an adequate aperture for HEB with dipoles of the same di. ameter instead of the original design specification of $7 \mathrm{~cm}$. Since binning requires a space in each dipcle for the trim sextupole winding, the effectiveness of magnet sorting is especially relevant to the important question raised at the SSC Laboratory. Expected use of sorting rather than binning is implied in a statement in ref. [5]: "The values of random b2 are $1 / 4$ of the scaled values. It is assumed that the dipoles will be sorted to bring about this reduction." The purpose of the present work is to confirm the validity of this assumption in the presence of potentially harmful effects that might affect the effectiveness of sorting.

Although it has not been studied by us so far, one crucial question for HEB is whether such a reduction factor could be expected independent of the magnitude of random errors. With $5 \mathrm{~cm}$ diameter, the expected $\mathrm{ms}$ value of random $b_{2}$ is 1.36 (E-4) $\mathrm{cm}^{-2}$ which is assumed to be reduced to 0.34 . The scaling used in ref. [5] reduces the rms value down to 0.73 when the diameter is increased to $7 \mathrm{~cm}$ and this will be further reduced to 0.18 , the value assumed in ref. [5] for the study of dynamic apertures, if the same reduction factor of four could be achieved by sorting. This is by no means obvious. Aside from the possible dependence of the reduction factor on the magnitude of random errors the effective ms value of 0.18 is approaching the expected achievable accuracy of measurements. In particular, it will be difficult to guarantee such an accuracy from warr.1 measurements alone. 


\section{TRACKING}

\section{A. HEB Lattice and Random Multipole Components}

The lattice consists of two nearly circular arcs containing 384 dipoles in 96 normal cells and 48 dipoles in 24 dispersion suppressing cells. Two arcs are connected by two long-straight sections. Each normal cell, some $78 \mathrm{~m}$ long with four dipoles, has a phase advance of $90^{\circ}$ in both traverse directions, resulting in the nominal design tunes of $34.42(\mathrm{H})$ and $33.38(\mathrm{~V})$. The maximum values of beta and dispersion in normal cells are $132 \mathrm{~m}$ and $3 \mathrm{~m}$, respectively. Initially, $b_{2}$ to $b_{6}$ and $a_{2}$ to $a_{6}$ were all taken into account in tracking. After finding out that the change in the dynamic aperture (defined here for 2,000 turns) or the smears due to higher multipoles is insignificant, we have taken only $b_{2}$ and $a_{2}$ for most of the calculations. As is usually the case for this type of tracking [5], random normal $\left(b_{1}\right)$ and skew $\left(a_{1}\right)$ quadrupole components were set to zero. Furthermore, the chromaticity correcting sextupoles are assumed to be unimportant in limiting the dynamic aperture.

\section{B. Sorting Procedures}

In its original form, Gluckstern-Ohnuma scheme sorts each time a group of magnets covering the phase advance of $360^{\circ}$. For HEB, this means we sort 16 magnets at a time, a number too few to be comfortable with. The procedure was therefore extended to 32 magnets at a time covering $720^{\circ}$. Sortings based on $b_{2}$ alone are designated as $S_{1}$ while $S_{2}$ denotes two-parameter sortings $[3,4]$ with both $b_{2}$ and $a_{2}$ as the sorting parameters. In the actual implementation of this sorting scheme, the choice of $S_{1}$ or $S_{2}$ will depend on the magnitude of $a_{2}$ variation. The rms value of 0.41 used in the present exercise may be near the lower boundary of the meaningful two-parameter sorting.

\section{Tracking}

Once an arrangement of dipoles is chosen, one particle with certain initial amplitudes $\left(x_{0} / V \beta_{x}=y_{0} /{ }^{\prime} \beta_{y}, x_{0}^{\prime}=y_{0}^{\prime}=0\right)$ is tracked, typically 500 turns, to estimate "smears" as a function of the initial amplitudes. If the particle is lost in less than 500 turns, the tracking is extended to 2.000 turns in order to find the dynamic aperture. As the variation of $B$ within each magnet is nontrivial, sextupole kicks are applied at three locations in a nagnet with the properly chosen ratio. There are at least two shortcomings in this tracking study compared with the one in ref. [5]: the number of turns $(2,000 \mathrm{vs}$
10,000 turns or more) and the choice of particles tracked (one particle at a time $v s$ "hundreds with well distributed initial displacements"). Other simplifying features used in our tracking are replacement of longstraight insertions and dispersion suppressors with a linear matched transfer matrix of the same phase advance, and no chromaticities. It is hoped that these simplifications are allowed in relative comparisons of sorted $v s$ random arrangements and of sorting $v s$ binning; the absolute magnitudes of the dynamic aperture are regarded at best as "semi-quantitative".

\section{RESULTS AND COMMENTS}

Main results from oui study are summarized in Figs. 1-6 showing smears as a function of the initial ampliture and the dynamic aperture under various conditions. The initial amplitude $x_{0}$ is at $B_{x}=106 \mathrm{~m}$. Since $B_{y}$ is $29.6 \mathrm{~m}$ at that location, the initial vertical

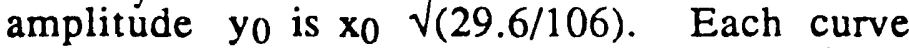
represents the smear and it is terminated with * symbol at the dynamic aperture. Five random arrangements were generated from one predetermined set of 384 magnets and this set was used for sorted arrangements as well. In order to simulate binning, $\sigma\left(b_{2}\right)$ was simply reduced by a factor of four without changing the magnet arrangement in each of five random cases.

Fig. 1 Five random arrangements and two sorted arrangements with $\sigma\left(b_{2}\right)=1.36(E-4) / \mathrm{cm}^{2}$ and $\sigma\left(a_{2}\right)=0.41(E-4) / \mathrm{cm}^{2}$. The advantage of two-parameter sorting $S_{2}$ over one-parameter sorting $S_{1}$ is questionable.

Fig. 2 Simulation of $a b_{2}$-binning. $S\left(a_{2}\right)$ is the sorted arrangement with $a_{2}$ as the sorting parameter. There is no advantage in sorting for this case.

Comparing the results in Fig. 2 with $\mathrm{S} 1$ and S2 in Fig. 1, one can claim that the sorting is somewhat better than the reduction of $\sigma$ (b2) by a factor of four although the difference is not significant.

Fig. 3 The working point is changed to $(34.58,33.62)$ from the nominal design of $(34.42,33.38)$. The performance of sorted arrangements deteriorates but still comparable to the binning. Fig. $4 \& 5$ With the effect of momentum dispersion, $(\Delta \mathrm{p} / \mathrm{p})=-0.001$ and +0.001 , respectively. This corresponds to $4 \sigma$ of the expected momentum distribution. The deterioration of $S_{1}$ performance with $(\Delta \mathrm{p} / \mathrm{p})=+0.001$ should be further investigated.

Fig. 6 With closed orbit distortion in both directions; $\pm 1 \mathrm{~mm}$ max. 34th and 33rd harmonic variations plus $\pm 0.5 \mathrm{~mm} \max$. random misalignments of diapoles. 


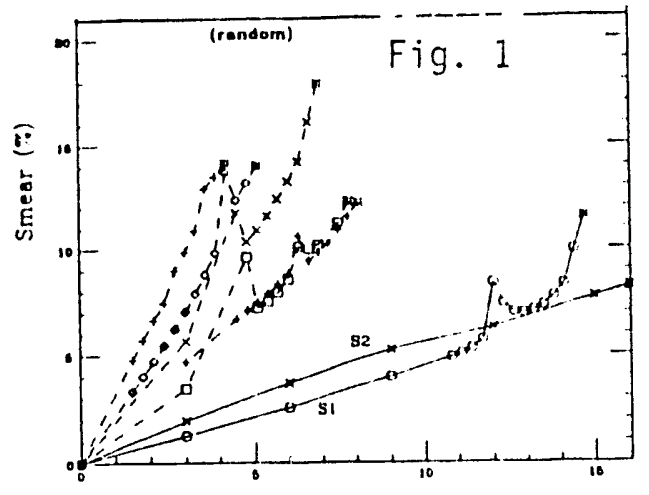

Initial Amplitude $X_{O}(\mathrm{~mm})$
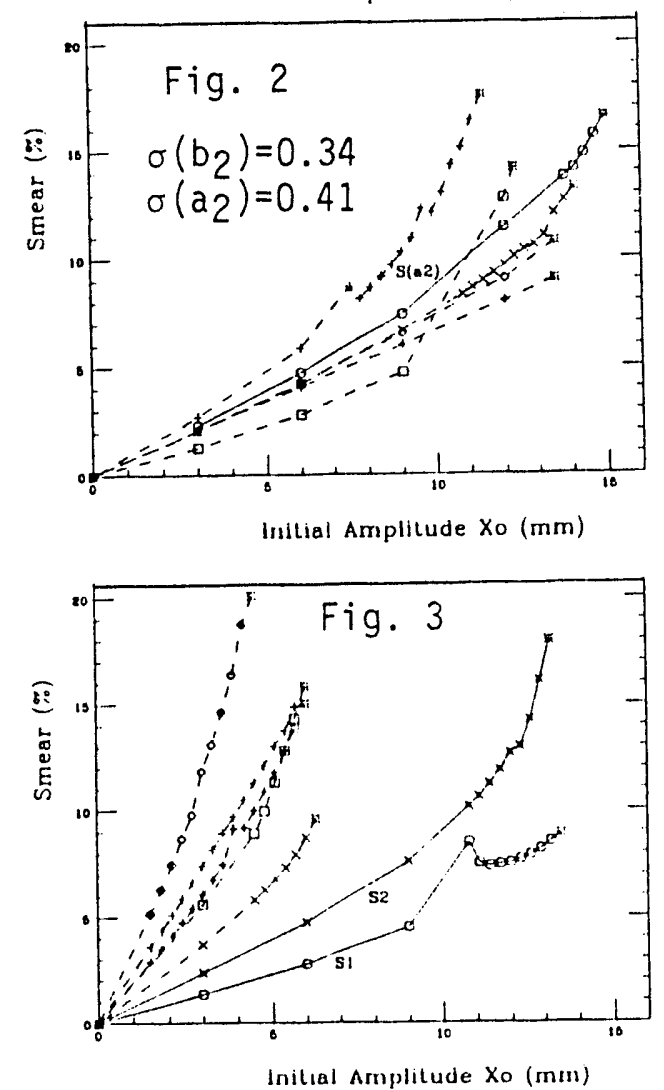
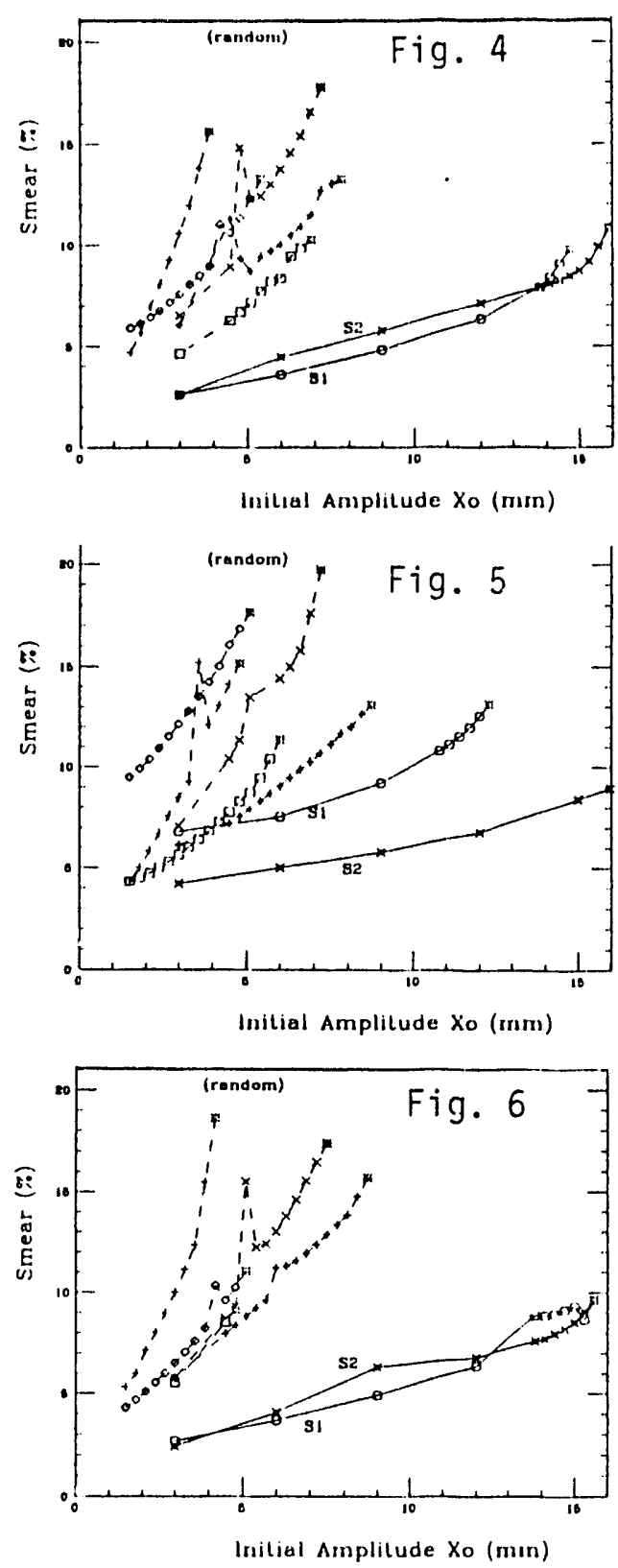

\section{REFERENCES}

[1] Richard Talman, "Field Trimming of SSC Dipoles," in Proceedings of the 1986 Summer Study on the Physics of the Superconducint Super Collider, p. $604,1986$.

[2] R.L. Gluckstem and S. Ohnuma, "Reduction of Sextupole distortion by Shuffling Magnets in Small Groups," IEEE Trans. Nucl. Sci., NS-32, P. 2314, 1985.

[3] Mingyang Li, "A Study of Nonlinear Motions in Large Synchrotrons," University of
Houston, Houston, Texas, Ph.D. Dissertation, 1990.

[4] Mingyang Li and Shoroku Ohnuma, "TwoParameter Sorting of Dipoles in Large Synchrotrons," in Proceedings of the 2nd European Particle Accelerator Conference, Nice-France, June 1990, vol. 2, p. 1175.

[5] A.W. Chao, S.K. Dutt, D.E. Johnson, T. Sen, and Y. Yan, "Dynamic Aperture and Extraction Studies for the SSC High Energy Booster," SSC Laboratory Report SSCL-296, September 1990. 

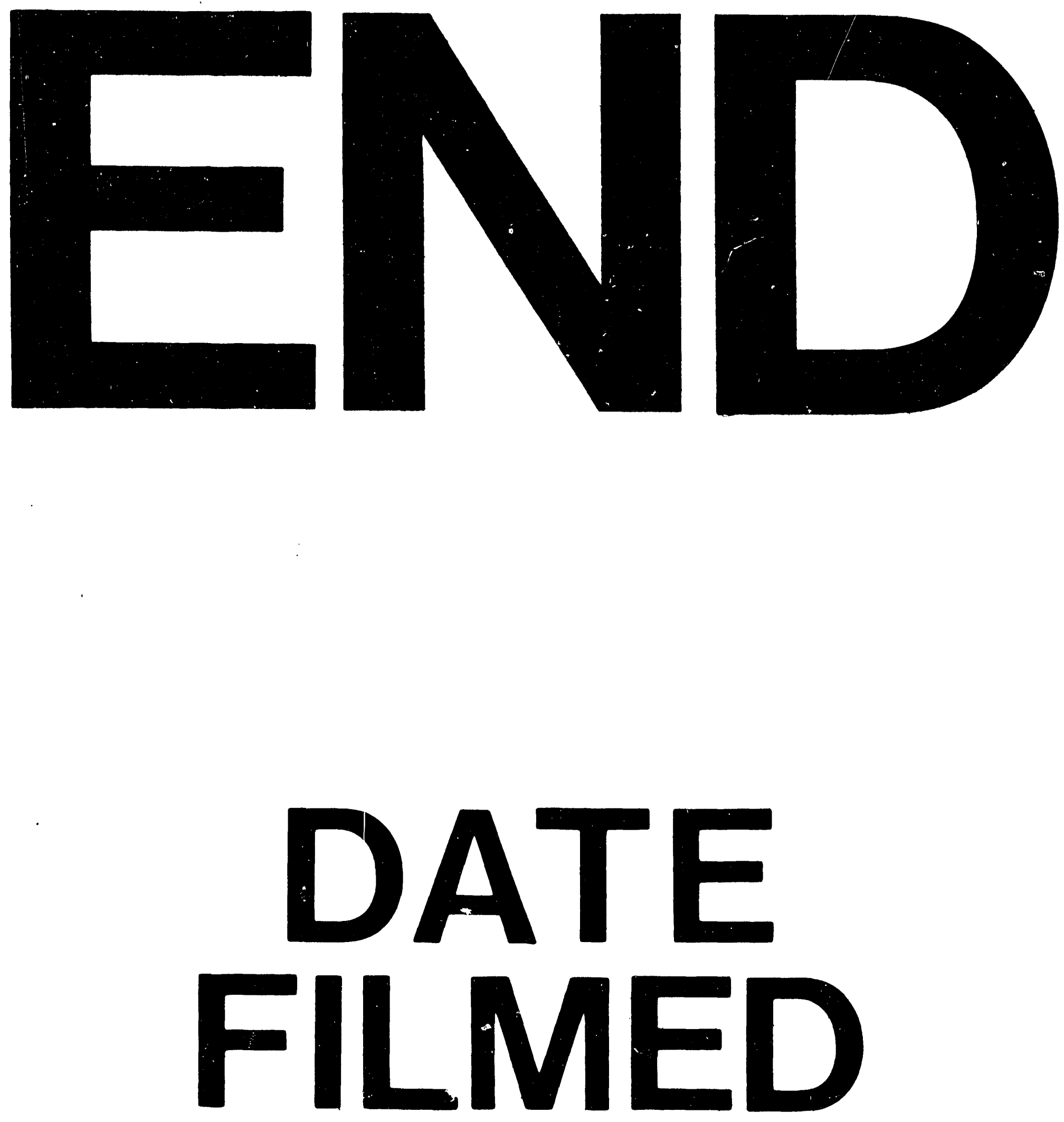

I

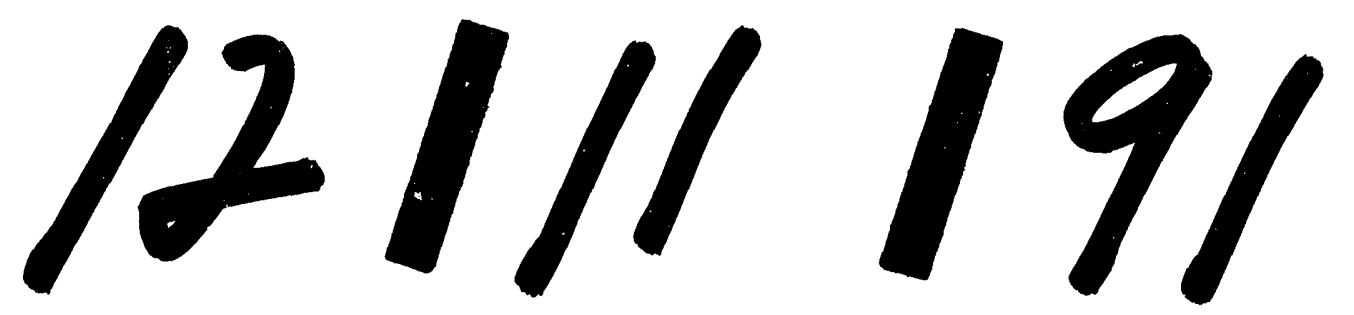

$\bar{I}$ 
